\title{
A Cascade MPC Control Structure for PMSM with Speed Ripple Minimization
}

\begin{abstract}
This paper addresses the problem of reducing the impact of periodic disturbances arising from the current sensor offset error on the speed control of a PMSM. The new results are based on a cascade model predictive control scheme with embedded disturbance model, where the per unit model is utilized to improve the numerical condition of the scheme. Results from an experimental application are given to support the design.
\end{abstract}

\section{INTRODUCTION}

Permanent Magnet Synchronous Motors (PMSMs) have been broadly adopted for industrial speed control applications due to their low volume and high efficiency. One of the most conventional control schemes for a PMSM is a cascade structure known as Field Oriented Control (FOC), where both inner and outer loop controllers are Proportional plus Integral (PI). The inner loop controller regulates the currents in the d-q rotating reference frame and its outer loop counterpart regulates the speed by providing the $\mathrm{q}$-axis reference current for the inner loop. This structure is mostly applied to a PMSM machine to maintain constant speed, but the speed control performance is degraded as a result of the pulsating torque [?] existing in the motor drive.

Pulsating torque in a PMSM is usually produced by various sources, such as cogging torque, flux harmonics and current sensor errors, and consists of the 1st, 2nd, 6th and 12th harmonics [?] where the fundamental is the synchronous frequency. The high frequency ripples could be removed by the load inertia or bandwidth of speed control loop, while the low frequency ripples that occur within the bandwidth could still cause the speed oscillation. Among these, the 1st harmonic is often the major cause of poor control performance due to the sensor offset error. Without suitable compensation for its effects, the speed will oscillate in the steady-state, especially at low values.

A number of controller design methods has been proposed to suppress the torque disturbance and/or the resulting speed ripples. These methods can be categorized into two groups: feedforward compensation and Internal Model Principle (IMP) approaches [?], respectively. For example, in [?] a feedforward approach is employed to calculate the torque ripples from the feedback errors and feeds forward the correction to cancel the disturbance on the q-axis current. A sizable body of literature addresses the periodic disturbance based on the IMP, which states that in order to follow a periodic reference or reject a periodic disturbance, the generator for the reference/disturbance must be included in the stable closed-loop control system. For example, [?] designs a robust controller based on IMP while [?] and [?] uses Iterative Learning Control (ILC).

Another possible candidate for electrical drives is Model Predictive Control (MPC), which is an optimization-based approach where the current control applied is obtained by minimizing the difference between the predicted behavior of system and its desired performance. MPC has long been established in process control since such applications can often afford a relatively long sample time to solve the Quadratic Programming (QP) problem that arises in the resulting algorithm on-line. Conversely, MPC is relatively less commonly encountered in electric drives and power electronics due to their fast sampling requirement and the computational load of the QP. In more recent years, with the development of faster micro-controllers and advances in MPC research, there has been an increasing trend to investigate the new control schemes for the applications of electric drives.

One popular topic is called Predictive Current Control (PCC) [?] [?] [?] that takes the advantage of the inherent features of the inverter. There are only finite possible switching states by turning on and off the gates for each leg of inverter. The underlying idea of PCC is that one or two-step prediction $\left(N_{p}=1\right.$ or 2$)$ of stator current is carried out for each of the possible switching states, and consequently the switching state that minimizes the cost function is selected as the input for the machine. Depending on the application, the cost function may consist of the distance between the prediction and reference vector, number of switches per cycle, power losses and other factors [?]. Since only finite choices available for the input, more precisely, PCC is termed as Finite Control Set MPC (FCS-MPC) in some literatures [?] [?]. Several different schemes of PCC have been invented with their performance analysed and compared in [?]. In general, the main features of PCC are the fast dynamic response, ability to impose constraints and possible absence of a modulator.

PCC for power converters and electric drives differs from the conventional MPC in the literatures [?] [?] [?], where longer prediction horizon is employed and the optimal inputs are solved under system constraints 
for the multivariable linear system. The application of the conventional MPC has been reported for Induction Motor (IM) [?] and PMSM [?]. In [?], a combination of speed and current control in a single controller is applied to full order electromechanical model of PMSM in $d-q$ reference frame. The constraints are imposed and the resulting input voltage vector needs to be implemented by the modulator. To tackle the problem of unmeasured disturbance, an extra integrator is superimposed on the MPC to remove steadystate error. [?] presents an MPC for the current loop with the estimation of disturbances using Recursive Least Square (RLS) that were fed forward for compensation. It requires the knowledge of steady-state values because the integrator is not embedded into the design. More lately, Predictive Functional Control (PFC), a type of MPC where the input is modelled by basis functions, has been utilized in the speed control of PMSM [?]. By taking the advantage of cascade structure, PFC is accompanied with an Extended State Observer (ESO) to compensate the impact of disturbances. The disturbance rejection in [?] and [?] can be categorized into the disturbance observer approach.

This paper develops a cascade MPC structure for a PMSM with current and speed control as the inner and outer loops, respectively. The inner current control loop is designed based on the principle of receding horizon control using the linearized per unit model of the PMSM. The outer loop is also an MPC with speed reference as the set-point signal and reference of q-axis current as the control signal. In order to improve speed control under sinusoidal disturbances due to the current sensor offset errors, the corresponding disturbance frequency modes are embedded in the design of outer loop MPC. This is different from the disturbance observer approach, where the disturbance is typically estimated by an observer and then canceled by subtracting it from the control signal. An additional benefit of the proposed approach in this paper is that with the embedded disturbance model, the implementation of MPC in real-time is greatly simplified as the steady-state information about the plant is no longer required. A more general treatment of this approach is termed Repetitive-Predictive Control [?]. In comparison with ILC approaches [?] [?] that use all frequency components, the proposed approach only embeds dominant frequencies such as zero and first frequencies into the design of MPC, which effectively reduces the closed-loop bandwidth and enhances the robustness properties in the presence of measurement noise and unmodelled dynamics [?].

This paper is organized as follows. Section II analyzes the source of speed ripples due to the measurement offset error from the sensor. Section III introduces the signal generator for constant and sinusoidal disturbances. Section IV gives the MPC design with different frequency modes embedded and Section ?? designs the cascade MPC control structure for inner and outer loop systems, respectively. Section VI presents the results from experimental implementation of the control structure on an industry-sized PMSM, together with frequency domain analysis. Section VII concludes the paper .

\section{BACKGROUND}

\section{A. PMSM Model}

The commonly used d-q model of a PMSM is expressed in terms of its rotor reference frame as

$$
\begin{aligned}
\dot{i_{d}} & =\frac{1}{L_{d}}\left(v_{d}-R_{s} i_{d}+\omega_{e} L_{q} i_{q}\right) \\
\dot{i_{q}} & =\frac{1}{L_{q}}\left(v_{q}-R_{s} i_{q}-\omega_{e} L_{d} i_{d}-\omega_{e} \phi_{m g}\right) \\
\dot{\omega}_{e} & =\frac{p}{J}\left(T_{e}-\frac{B}{p} \omega_{e}-T_{L}\right) \\
T_{e} & =\frac{3}{2} p\left[\phi_{m g} i_{q}+\left(L_{d}-L_{q}\right) i_{d} i_{q}\right]
\end{aligned}
$$

where $\omega_{e}$ is the electrical speed and is related to the rotor speed by $\omega_{e}=p \omega_{m}$ with $p$ noting the number of pole pairs, $\omega_{m}$ is the mechanical speed, $v_{d}$ and $v_{q}$ denote the stator voltages in the $\mathrm{d}$-q frame, $i_{d}$ and $i_{q}$ denote the stator currents in the d-q frame and $T_{L}$ is load torque.

For a surface mounted PMSM where effect of saliency is negligible, the d-axis inductance is equal to the q-axis inductance $\left(L_{d}=L_{q}\right)$ and hence there is no reluctance torque component $\left(\left(L_{d}-L_{q}\right) i_{d} i_{q}=0\right)$. As a result, the torque in (4) is produced only by the interaction of permanent magnet flux linkage and q-axis current component, as described by (5). In addition, vector control in the d-q frame usually sets the reference of $\mathrm{d}$-axis current to be zero $\left(i_{d}^{*}=0\right)$ except that in the field weakening region. With a welldesigned current controller, the d-axis current $i_{d}$ is expected to follow $i_{d}^{*}=0$. Under this assumption, the torque can also be approximated by (5), although it is not a necessary condition for surface mounted PMSM.

$$
T_{e}=\frac{3}{2} p \phi_{m g} i_{q}
$$

Substituting (5) into (3) gives,

$$
\dot{\omega}_{e}=\frac{p}{J}\left(\frac{3}{2} p \phi_{m g} i_{q}-\frac{B}{p} \omega_{e}-T_{L}\right)
$$

\section{B. Current sensor offset error}

The idea of vector control is to transform the three phase ac currents $i_{a}, i_{b}$ and $i_{c}$ into their representation, $i_{d}$ and $i_{q}$, in the rotor reference frame

$$
\left[\begin{array}{c}
i_{d} \\
i_{q} \\
i_{0}
\end{array}\right]=\frac{2}{3} T_{a b c}\left[\begin{array}{c}
i_{a} \\
i_{b} \\
i_{c}
\end{array}\right]
$$


where

$T_{a b c}=\left[\begin{array}{ccc}\sin \left(\theta_{e}+\frac{\pi}{2}\right) & \sin \left(\theta_{e}-\frac{\pi}{6}\right) & \sin \left(\theta_{e}+\frac{7 \pi}{6}\right) \\ \cos \left(\theta_{e}+\frac{\pi}{2}\right) & \cos \left(\theta_{e}-\frac{\pi}{6}\right) & \cos \left(\theta_{e}+\frac{7 \pi}{6}\right) \\ \frac{1}{2} & \frac{1}{2} & \frac{1}{2}\end{array}\right]$

Here, $\theta_{e}=\int \omega_{e}(t) d t$ denotes the electrical angle of PMSM. After transformation, the $i_{d}$ and $i_{q}$ components are taken as dc values and the controllers are designed to regulate them to their desired values, which mimics the control principles of dc motors. The values of the three phase ac current components are usually obtained by two current transducers and then digitalized by A/D converters, which produces a dc offset voltage superimposed on the scaled sinusoidal signal. The dc offset varies unpredictably due to the thermal effect of analog devices, thus making it difficult to separate the unwanted dc offset from the sinusoidal measurement [?]. As a result the measured sinusoidal currents have non-zero dc offsets and this phenomenon can be modeled as

$$
\begin{aligned}
i_{a}^{\prime} & =i_{a}+\Delta i_{a} \\
i_{b}^{\prime} & =i_{b}+\Delta i_{b} \\
i_{c}^{\prime} & =-\left(i_{a}+i_{b}\right)-\left(\Delta i_{a}+\Delta i_{b}\right) .
\end{aligned}
$$

where $i_{a}$ and $i_{b}$ are the actual three phase currents, $i_{a}^{\prime}$, $i_{b}^{\prime}$ and $i_{c}^{\prime}$ are measured current values contaminated by the unwanted dc offset errors and $\Delta i_{a}$ and $\Delta i_{b}$ are DC offset errors.

Application of the abc/dq transformation (7) to the contaminated three phase currents of (8) results in the addition of sinusoidal oscillations to the actual $d-q$ axis current [?]

$$
i_{q}^{\prime}=i_{q}+\Delta i_{q}, \quad i_{d}^{\prime}=i_{d}+\Delta i_{d}
$$

where $i_{q}$ and $i_{d}$ denote the actual d-q axis currents and $\Delta i_{d}$ and $\Delta i_{q}$ are the sinusoidal disturbances due to the offset errors $\Delta i_{a}$ and $\Delta i_{b}$, respectively

$$
\begin{aligned}
& \Delta i_{q}=\Delta I \cos \left(\theta_{e}+\varphi\right) \\
& \Delta i_{d}=\Delta I \sin \left(\theta_{e}+\varphi\right)
\end{aligned}
$$

where

$$
\begin{aligned}
& \Delta I=\frac{2}{\sqrt{3}} \sqrt{\left(\Delta i_{a}^{2}+\Delta i_{a} \Delta i_{b}+\Delta i_{b}^{2}\right)}, \\
& \varphi=\tan ^{-1}\left(\frac{\sqrt{3} \Delta i_{a}}{\Delta i_{a}+2 \Delta i_{b}}\right) .
\end{aligned}
$$

Also from (9) and (10) the frequency of the disturbance oscillations is the same as the electrical speed of PMSM. In the steady-state when the motor is running at constant speed, the frequency of oscillation is fixed. Fig. 1 shows the case where the motor used for controller design and experimental testing in this paper is running at $300 \mathrm{rpm}$ and the oscillation due to the offset error is of frequency $10 \mathrm{~Hz}$. Here, the disturbance was generated using current bias error of 0.48 Am for illustration purpose.

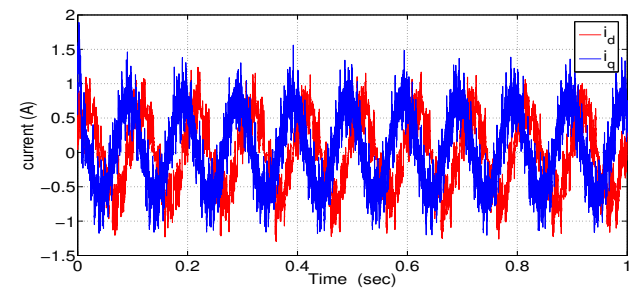

Fig. 1. Experiment: oscillation of $i_{d}$ and $i_{q}$ due to dc offset error.

\section{Speed ripple}

Using the electrical and mechanical models (1), (2) and (6), respectively, of a PMSM in the d-q frame, the cascade structure, shown in Fig. 2 has been widely used for closed-loop speed control, where $\omega_{m}^{*}$ denotes the constant rotor speed demand. In this case the sinusoidal disturbance, $\Delta i_{d}$ and $\Delta i_{q}$, caused by the measurement errors can be equivalently treated as an external disturbance entering the closed-loop while assuming that $i_{d}$ and $i_{q}$ are error free. If the frequency

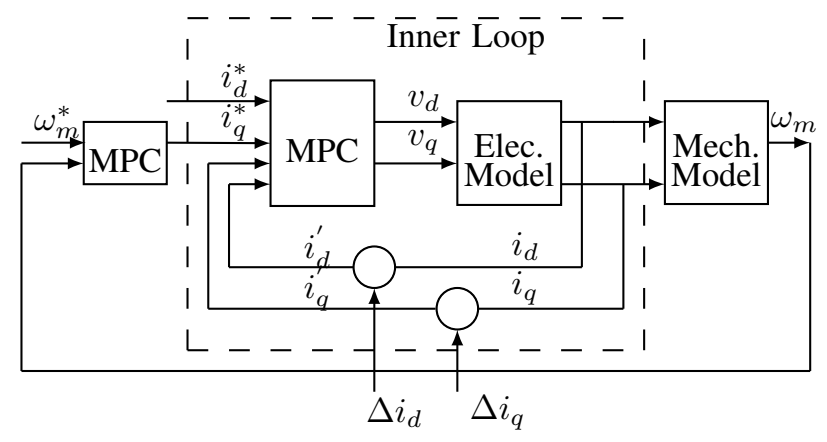

Fig. 2. Cascade MPC scheme.

of disturbance is within the bandwidth of the speed loop, the steady-state speed will oscillate at the same frequency as disturbance in the absence of suitable compensation.

\section{The Signal Generator}

The discussion of the previous section establishes that the frequency of the steady-state speed oscillation can be accurately determined once speed reference is a-priori known. Hence this frequency information can be used in the MPC design to reject the disturbance. In this paper design is undertaken using the RepetitivePredictive Controller (RPC) design method [?] [?]. The remainder of this section gives the relevant background.

\section{A. Embedded Signal Generator}

By the Internal Model Principle (IMP) [?], to follow a reference signal and reject a disturbance with 
zero steady-state error, the generating polynomial of the reference and disturbance has to be embedded into the controller. Consider, for example, the case when the reference signal for an application contains multiple frequencies. Then the resulting generating polynomial will contain all periodic modes, and the number of these is proportional to the period of reference/disturbance signal and inversely proportional to the sampling interval. The result could be a very high order control system, especially under fast sampling, and hence the possibility of numerical sensitivity, noise amplification, sensitivity to modeling errors, and other undesirable problems in practical applications.

An alternative to including all the periodic modes is to embed fewer periodic modes at a given instance [?], where the frequency components of a given signal are analyzed and its reconstruction performed using a frequency sampling filter model, from which the significant frequencies are identified and error analysis is used to justify the selections. Once the significant frequency components have been selected, the generating polynomial is available and the design proceeds by first augmenting the plant state-space model with the modes selected from the frequency response of the reference signal. Receding horizon control applied to this augmented model results in a feedback controller for the resulting state-space model, and the extension to also include disturbance rejection is immediate.

In this work the generating polynomial is formulated in the $z$-transform domain. For example, in the case of a constant reference signal

$$
D_{r}(z)=1-z^{-1}
$$

Note that $D_{r}(z)$ is the denominator of the z-transform of constant signals and also denotes the denominator of the integrator used in a discrete-time feedback controller. Since $D_{r}(z)$ only contains the polynomial for constant signals, it is also referred as zero frequency mode.

In [?], the generating polynomial of a general periodic signal is derived from a frequency sampling filter decomposition, which could contain multiple dominant frequency modes. Particular to the requirements in the PMSM application of tracking a constant reference speed and rejecting the sinusoidal disturbance and constant load torque disturbance, the signal generator is

$$
\begin{aligned}
D(z) & =\left(1-z^{-1}\right)\left(1-e^{j \omega} z^{-1}\right)\left(1-e^{-j \omega} z^{-1}\right) \\
& =\left(1-z^{-1}\right)\left(1-2 \cos (\omega) z^{-1}+z^{-2}\right) \\
& =1+d_{1} z^{-1}+d_{2} z^{-2}+d_{3} z^{-3}
\end{aligned}
$$

where $\omega=\frac{2 \pi}{M}$ denotes the fundamental frequency and $M$ is the number of samples in each period. Note that in (13), zero frequency component $1-z^{-1}$ is always included to compensate constant component in the reference and disturbance signal. In addition, the component $1-\cos (\omega) z^{-1}+z^{-2}$, also referred as 1 st frequency mode, is the denominator of z-transform of the sinusoidal signal with fundamental frequency $\omega$. By combining these two terms, $D(z)$ contains both zero and $1 s t$ frequency modes.

For example, if the motor is running at $n=300 \mathrm{rpm}$ in the steady-state and the sampling period is $T_{s}=$ $200(\mu s)$, the period of disturbance due to the offset error is

$$
T_{p}=\frac{60}{n \cdot p}=\frac{60}{300 \times 2}=0.1(\mathrm{sec})
$$

where the physical unit rpm is converted to electrical speed with the pair of poles equal to 2 . The number of samples for one period of the disturbance is calculated using

$$
M=\frac{T_{p}}{T_{s}}=\frac{0.1}{2 \times 10^{-4}}=500(\text { samples })
$$

with fundamental frequency

$$
\omega=\frac{2 \pi}{M}=0.0126(\mathrm{rad} / \text { sample }) .
$$

In this case

$$
D(z)=1-2.9998 z^{-1}+2.9998 z^{-2}-z^{-3}
$$

\section{CASCADE MPC With EMBEDded SignaL GENERATORS}

This section presents how to embed the signal generators into the design of cascade MPC structure, assuming that the control objective is to follow a constant speed reference signal while minimizing the effect of sinusoidal disturbance caused by the measurement error from current sensors.

As illustrated in Fig. 2, the scheme considered in this paper uses MPC as the basis for both the inner and outer loop controllers. It is a common practice that the design of a cascade control system begins at the inner loop controller, followed by the outer loop control system. The controller structure for the inner loop is often simpler than the one used in the outerloop system. Since both controllers will be designed using receding horizon control principle, the difference between the controller structures is related to the signal generator that is embedded into the design model, as explained in the sequel.

\section{A. The Design Model for Inner Loop MPC}

The inner loop MPC uses the electrical model described by (1) and (2), which is a nonlinear and coupled multivariable system. Here, the control signals are $v_{d}$ and $v_{q}$, and the output signals are $i_{d}$ and $i_{q}$. First step in the design of inner-loop controller is to obtain the 
linearized model for the $\mathrm{d}-\mathrm{q}$ axis current, which has the form:

$$
\begin{aligned}
& {\left[\begin{array}{c}
\dot{i}_{d} \\
\dot{i}_{q}
\end{array}\right]=\left[\begin{array}{cc}
-\frac{R_{s}}{L_{d}} & \frac{L_{q}}{L_{d}} \omega_{e 0} \\
-\frac{L_{d}}{L_{q}} \omega_{e 0} & -\frac{R_{s}}{L_{q}}
\end{array}\right]\left[\begin{array}{l}
i_{d} \\
i_{q}
\end{array}\right]} \\
& +\left[\begin{array}{cc}
\frac{1}{L_{d}} & 0 \\
0 & \frac{1}{L_{q}}
\end{array}\right]\left[\begin{array}{c}
v_{d} \\
v_{q}
\end{array}\right] \\
& +\left[\begin{array}{c}
\frac{L_{q}}{L_{d}}\left(\omega_{e 0} i_{q 0}+i_{q 0} \omega_{e}\right) \\
-\frac{\phi_{m g} \omega_{e}}{L_{q}}-\frac{L_{d}}{L_{q}}\left(\omega_{e 0} i_{d 0}+i_{d 0} \omega_{e}\right)
\end{array}\right]
\end{aligned}
$$

where the linearization is performed around the steadystate operating condition defined by the parameters $\omega_{e 0}, i_{d 0}$ and $i_{q 0}$. Note that all the state and input variables in (14) represents the deviation variables from the steady state values. The last column in (14) represents the disturbances to the inner loop control system.

Denoting

$$
A_{c}=\left[\begin{array}{cc}
-\frac{R_{s}}{L_{d}} & \frac{L_{q}}{L_{d}} \omega_{e 0} \\
-\frac{L_{d}}{L_{q}} \omega_{e 0} & -\frac{R_{s}}{L_{q}}
\end{array}\right] ; B_{c}=\left[\begin{array}{cc}
\frac{1}{L_{d}} & 0 \\
0 & \frac{1}{L_{q}}
\end{array}\right],
$$

and assuming a zero-order hold and a sampling interval $T_{s}$, discretization of the continuous-time state space model (14) leads to the discrete-time state space model:

$$
\begin{aligned}
x_{m}(k+1) & =A_{m} x_{m}(k)+B_{m} u(k)+\Omega_{m} \mu(k) \\
y(k) & =C_{m} x_{m}(k)
\end{aligned}
$$

where $x_{m}(k)=y(k)=\left[i_{d}(k) i_{q}(k)\right]^{T}, u(k)=$ $\left[v_{d}(k) v_{q}(k)\right]^{T}, A_{m}=e^{A_{c} T_{s}}, \quad B_{m}=\left(\int_{0}^{T_{s}} e^{A_{c} \tau} d \tau\right)$ and $C_{m}$ is the identity matrix with dimension of $2 \times 2$. $\Omega_{m} \mu(k)$ represents the discretized disturbance term.

The primary roles of inner loop control system are to reject the disturbances as fast as possible and to overcome the nonlinearity and parameter uncertainties using feedback control. Meanwhile, the accuracy in the steady-state operation is a less important factor when designing an inner loop control system. From this point of view, integral action may not be necessary for the inner loop MPC design. However, as shown later in the section, the simplicity of implementation of the real-time control system partially justifies embedding integrators into the design of MPC for the inner loops in which all the variables will be expressed as incremental variables, hence the information concerning the steady-state operation such as the parameters $i_{d 0}, i_{q 0}$, $v_{d 0}$ and $v_{q 0}$ will not be required in the implementation.

How to embedding integrators into MPC is discussed in [?]. In essence, this procedure is to embed the polynomial of the integrator (see (12)) into the state-space model given by (15) and (16), as discussed briefly below. Taking a difference operation on both sides of (15), equivalent to multiplication of $D_{r}\left(q^{-1}\right)$ on both sides, leads to

$$
\begin{aligned}
& x_{m}(k+1)-x_{m}(k)=A_{m}\left(x_{m}(k)-x_{m}(k-1)\right) \\
& \quad+B_{m}(u(k)-u(k-1))+\Omega_{m}(\mu(k)-\mu(k-1)),
\end{aligned}
$$

which is

$\Delta x_{m}(k+1)=A_{m} \Delta x_{m}(k)+B_{m} \Delta u(k)+\Omega_{m} \Delta \mu(k)$

where

$$
\begin{aligned}
& \Delta x_{m}(k+1)=x_{m}(k+1)-x_{m}(k), \\
& \Delta x_{m}(k)=x_{m}(k)-x_{m}(k-1), \\
& \Delta u(k)=u(k)-u(k-1) .
\end{aligned}
$$

These are the increments of the variables $x_{m}(k)$ and $u(k)$. By choosing a new state variable vector

$$
x(k)=\left[\begin{array}{ll}
\Delta x_{m}(k)^{T} & y(k)
\end{array}\right]^{T}
$$

, the design model for the inner loop control system is obtained as

$$
\begin{gathered}
\overbrace{\left[\begin{array}{c}
\Delta x_{m}(k+1) \\
y(k+1)
\end{array}\right]}^{x(k+1)}=\overbrace{\left[\begin{array}{cc}
A_{m} & o_{m} \\
C_{m} A_{m} & I_{2 \times 2}
\end{array}\right]}^{A} \overbrace{\left[\begin{array}{c}
\Delta x_{m}(k) \\
y(k)
\end{array}\right]}^{x(k)} \\
+\overbrace{\left[\begin{array}{c}
B_{m} \\
C_{m} B_{m}
\end{array}\right]}^{B} \Delta u(k)+\left[\begin{array}{c}
\Omega_{m} \\
C_{m} \Omega_{m}
\end{array}\right] \Delta \mu(k)
\end{gathered}
$$$$
y(k)=\overbrace{\left[\begin{array}{ll}
o_{m} & I_{2 \times 2}
\end{array}\right]}^{C}\left[\begin{array}{c}
\Delta x_{m}(k) \\
y(k)
\end{array}\right],
$$

where $I_{2 \times 2}$ is the identity matrix with dimension of $2 \times 2, o_{m}$ is a zero matrix with dimension of $2 \times 2$. From (14), the increment of the disturbance term, $\Delta \mu(k)$, mainly depends on the change of $\omega_{e}$. Thus, the disturbance term in (19) will become small when the variation of $\omega_{e}$ is reduced. In any case, closedloop feedback control will reduce the effect of the disturbance term.

Note that the formulation of the incremental variables $\Delta u(k)$ and $\Delta x_{m}(k)$ does not require their steady-state information. By adding and subtracting the steady-state value of input $\left(u_{s s}\right)$ on the right hand side of (18),

$$
\Delta u(k)=u(k)+u_{s s}-u(k-1)-u_{s s}
$$

the actual inner loop control signal can be calculated using the velocity form of the MPC

$$
u^{a c t}(k)=u^{a c t}(k-1)+\Delta u(k)
$$

where $u^{a c t}(k)=u(k)+u_{s s}$. By setting the initial sample of the control signal $u^{a c t}$ equal to the actual measurement of $v_{d}$ and $v_{q}$, based on (21), the actual $\mathrm{d}-\mathrm{q}$ axis currents can be calculated using the optimized $\Delta u(k)$ without their steady-state information. Furthermore, because the actual control signal is calculated 
using the previous sample of the same signal, when performing controller switching, bumpless transfer is guaranteed as demonstrated in the experimental results of this paper (see Section VI).

\section{B. The Design Model for Outer Loop MPC}

The dynamic relationship that links the inner loop with the outer loop is described by (6), where the input variable is $i_{q}$ and the output is $\omega_{e}$, and

$$
\dot{\omega}_{e}=\frac{p}{J}\left(\frac{3}{2} p \phi_{m g} i_{q}-\frac{B}{p} \omega_{e}-T_{L}\right)
$$

However, because the q-axis current $i_{q}$ is the output of the inner loop control system, it is not available for the manipulation in the outer loop. Instead, the free variable for the manipulation is the set-point signal to the $\mathrm{q}$-axis current, $i_{q}^{*}$. From the inner closed-loop system, the relationship between the output $i_{q}$ and the set-point signal $i_{q}^{*}$ is approximated by

$$
\dot{i_{q}}=-\frac{1}{\alpha} i_{q}+\frac{1}{\alpha} i_{q}^{*}
$$

where $\alpha$ is the time constant of first order approximation and its value could be obtained from the dominant pole of inner closed-loop. Note that the steady state gain of (23) is unity because of the existence of an integrator in the inner loop control system. The continuous time state-space model for the outer loop control system is formulated using (22) and (23), which is then discretized with sampling interval $T_{s}$. The key reason for using the continuous time model (23) to approximate the inner closed-loop system is because there is a difference between the sampling rates of inner and outer loops. Typically, the sampling rate for the inner loop is about twice as fast as the one chosen for the outer loop control. For a general expression, the discrete-time state space model is denoted by

$$
\begin{aligned}
x_{m}(k+1) & =A_{m} x_{m}(k)+B_{m} u(k)+\Omega_{m} \mu(k) \\
y(k) & =C_{m} x_{m}(k)
\end{aligned}
$$

where $x_{m}(k)=\left[i_{q}(k) \omega_{e}(k)\right]^{T}, u(k)=i_{q}^{*}(k)$ and $y(k)=\omega_{e}(k)$. The disturbance term $\mu(k)$ contains the load torque $T_{L}$ that is constant, and the sinusoidal disturbance with period $\omega$ caused by the sensor error. From Section III, the disturbance generation polynomial $D(z)$ is described by (13). How to embed the disturbance polynomial $D(z)$ into the outer loop model follows the procedure given in [?], which is briefly stated as below.

Let $D\left(q^{-1}\right)$ denote the shift operator form of $D(z)$ (see 13) and define the filtered state and input vectors $x_{s}(k)$ and $u_{s}(k)$, respectively, as

$$
x_{s}(k)=D\left(q^{-1}\right) x_{m}(k), u_{s}(k)=D\left(q^{-1}\right) u(k)
$$

Here, the filtered state and input vectors are obtained by applying the operator $D\left(q^{-1}\right)$ to the original state and input vectors. Since $D(z)$ contains all the frequencies of the disturbance $\mu(k)$, applying the operator $D\left(q^{-1}\right)$ to the disturbance $\mu(k)$ results

$$
D\left(q^{-1}\right) \mu(k) \approx 0
$$

Therefore, applying the operator $D\left(q^{-1}\right)$ to the state equation in (24) gives,

$$
x_{s}(k+1)=A_{m} x_{s}(k)+B_{m} u_{s}(k)
$$

Similarly, multiplying (25) by $D\left(q^{-1}\right)$ gives

$$
D\left(q^{-1}\right) y(k+1)=C_{m} A_{m} x_{s}(k)+C_{m} B_{m} u_{s}(k)
$$

By taking advantage of the shift operator $D\left(q^{-1}\right),(29)$ is replaced with the difference equation:

$$
\begin{aligned}
y(k+1) & =-d_{1} y(k)-d_{2} y(k-1)-d_{3} y(k-2) \\
& +C_{m} A_{m} x_{s}(k)+C_{m} B_{m} u_{s}(k)
\end{aligned}
$$

With the new state variable defined by

$$
x(k)=\left[\begin{array}{llll}
x_{s}^{T}(k) & y(k) & y(k-1) & y(k-2)
\end{array}\right]^{T}
$$

the design model for the outer loop control system is obtained:

$$
\begin{aligned}
x(k+1) & =A x(k)+B u_{s}(k) \\
y(k) & =C x(k)
\end{aligned}
$$

where the system matrices are given by,

$$
\begin{gathered}
A=\left[\begin{array}{cccc}
A_{m} & 0 & 0 & 0 \\
C_{m} A_{m} & -d_{1} & -d_{2} & -d_{3} \\
o_{m} & 1 & 0 & 0 \\
o_{m} & 0 & 1 & 0
\end{array}\right] \\
B=\left[\begin{array}{llll}
B_{m}^{T} & \left(C_{m} B_{m}\right)^{T} & 0 & 0
\end{array}\right]^{T} \\
C=\left[\begin{array}{llll}
o_{m} & 1 & 0 & 0
\end{array}\right] .
\end{gathered}
$$

$o_{m}$ is zero row vector with dimension $1 \times 2$.

After obtaining the filtered control signal as stated in the next section, the actual control signal $\left(i_{q}^{*}\right)$ will be reconstructed. Note that from (26), by adding and subtracting a constant $u_{s s}$, the following relationship is obtained:

$$
D\left(q^{-1}\right)\left(u(k)+u_{s s}-u_{s s}\right)=u_{s}(k)
$$

which is,

$$
D\left(q^{-1}\right) u^{a c t}(k)=u_{s}(k)
$$

where $u^{a c t}(k)=u(k)+u_{s s}$ and $D\left(q^{-1}\right) u_{s s}=0$ due to the difference term $1-q^{-1}$ contained in $D\left(q^{-1}\right)$. Expanding (33) leads to the computation of the actual control signal $u^{a c t}(k)$ :

$$
\begin{aligned}
u^{a c t}(k) & =u_{s}(k)-d_{1} u^{a c t}(k-1)-d_{2} u^{a c t}(k-2) \\
& -d_{3} u^{a c t}(k-3)
\end{aligned}
$$

This formulation of the actual control signal gives an advantage in the implementation of the MPC system, which does not require the steady-state information of the control signal. Particularly, when the controller structure changes, the past states of the actual control signal used in (33) guarantee a smooth transition of the control signal (called bumpless transfer), as demonstrated in the experimental results. 


\section{MPC Design}

The remaining task in the design of inner and outer MPC systems is to optimize the control signals $(\Delta u(k)$ for the inner loop and $u_{s}(k)$ for the outer loop). The details of the MPC design can be found in [?] [?]. For completeness of this paper, the design procedure is briefly stated as below. At sampling instant $k$, assuming a control horizon $N_{c}$ and prediction horizon $N_{p}$, the control objective for the inner loop MPC system is to find the optimal control input $\Delta u$ that minimizes the cost function

$$
\begin{gathered}
J=\sum_{i=1}^{N_{p}} x^{T}(k+i \mid k) Q_{i} x(k+i \mid k)+ \\
\sum_{j=0}^{N_{c}} \Delta u^{T}(j) R_{i} \Delta u(j)
\end{gathered}
$$

where the states and control are defined by the inner loop design model (see (19)), $Q_{i} \geq 0$ and $R_{i}>0$ are the weighting matrices. Similarly, for the outer loop MPC system, the control objective is to find the optimal control input $u_{s}(k)$ that minimizes the cost function

$$
\begin{aligned}
& J=\sum_{i=1}^{N_{p}} x^{T}(k+i \mid k) Q_{o} x(k+i \mid k)+ \\
& \sum_{j=0}^{N_{c}} u_{s}^{T}(j) R_{o} u_{s}(j)
\end{aligned}
$$

where the states and control are defined by the outer loop design model (see (31)). In this paper, both $Q_{o}$ and $Q_{i}$ are chosen as $C^{T} C$ with respective $C$ matrices from inner and outer loop models. $R_{i}$ and $R_{o}$ are tuned to reflect the demand of closed-loop response speed, for instance, the diagonal elements in $R_{i}$ are far smaller than the parameter $R_{o}$ so to ensure that the inner loop MPC has a much faster response speed than the outer loop control system.

One of the major strengths of MPC is the ability to impose constraints where, for example, in the case of the control input practically relevant constraints are of the form

$$
u^{\min } \leq u^{a c t}(k) \leq u^{\max }
$$

where $u^{\max }$ and $u^{\text {min }}$ are upper and lower limits for the control inputs. For the inner loop MPC, the constraints for the control signal are imposed via (21) as

$$
u^{\text {min }} \leq u^{a c t}(k-1)+\Delta u(k) \leq u^{\max }
$$

and for the outer loop MPC, the constraints for the control signal are imposed via (34) as

$$
\begin{aligned}
u^{\text {min }} \leq & u_{s}(k)-d_{1} u^{a c t}(k-1)-d_{2} u^{a c t}(k-2) \\
& -d_{3} u^{a c t}(k-3) \leq u^{\max }
\end{aligned}
$$

The essence to the solution of the constrained control problem is to minimize the cost function (35) subject to the inequality constraints (38) for the inner loop MPC and for the outer loop MPC, minimize the cost function (36) subject to the inequality constraints (39). The solution of the constrained minimization problem is found using Quadratic Programming algorithm in real-time [?]. When the unconstrained solution exceeds the constraints, the constraints become active, and MPC finds the optimal solution subject to constraints. The situation usually occurs at start-up and shutdown of PMSM. At the steady-state operation, the constraints in most cases are not active, thus, in essence, the MPC is equivalent to linear time invariant feedback control.

\section{Closed-loop input sensitivity function}

In the absence of constraints, the predictive control systems are linear time-invariant state feedback control systems. Their closed-loop control performances in the presence of input disturbance can be analyzed in terms of the input, or load, sensitivity function [?]. Since the closed-loop performance from the outer loop MPC determines the overall performance of the cascade MPC system in the presence of disturbance, the sensitivity function, particularly the input sensitivity function is calculated using the predictive controller without constraints.

Figure 3 shows the frequency response $G_{y d}\left(e^{j \omega}\right)$ of input sensitivity function for closed-loop control considered with only zero frequency mode (12) embedded and both the zero frequency and 1st frequency ( $\omega=0.0126)$ modes (13) embedded in the MPC design for different choices of the control weighting $R_{o}$ in the cost function. This sensitivity function is zero at the frequency of the disturbance and hence its effects on performance are rejected.

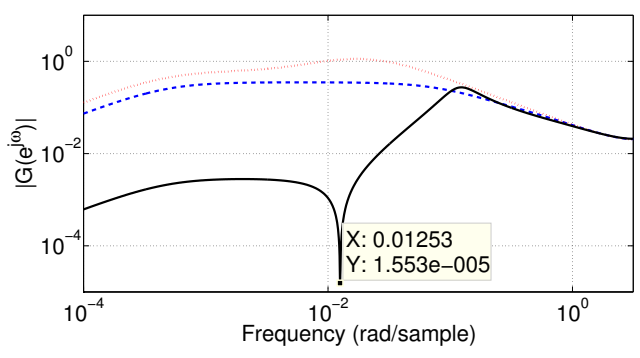

Fig. 3. Frequency response of input sensitivity function. Dot: zero frequency with $R_{o}=100$; Dash: zero frequency with $R_{o}=1$; Solid: zero and 1st frequency with $R_{o}=1000$.

\section{IMPLEMENTATION}

\section{A. Per Unit Model}

As MPC is a model based method, using the motor model of Section II in SI units would cause numerical problem when tuning the design. For example, using (3), a small inertia value in $\mathrm{kg} \cdot \mathrm{m}^{2}$ would lead to a very large coefficient $\left(\frac{p}{J}\right)$ for $T_{e}$ in $N m$ and hence the controller gain must be numerically very small. Hence it is more convenient to use the per unit model of the PMSM in MPC design. The base values of parameters and variables, as listed in Table I, are chosen as

$$
\begin{aligned}
& I_{b}=p P_{b} / U_{b}, \quad R_{b}=U_{b} / I_{b}, T_{b}=U_{b} I_{b} / \omega_{e b} \\
& \phi_{b}=U_{b} / \omega_{e b}, L_{b}=R_{b} / \omega_{e b} \\
& J_{b}=p P_{b} / \omega_{e b}^{2}, \quad B_{b}=T_{b} / \omega_{e b}
\end{aligned}
$$

Scaling the parameters and variables with their own base value, the model of Section II is replaced by its per unit version

$$
\begin{aligned}
& \dot{i_{d}}=\frac{\omega_{e b}}{L_{d}}\left(v_{d}-R_{s} i_{d}+\omega_{e} L_{q} i_{q}\right) \\
& \dot{i_{q}}=\frac{\omega_{e b}}{L_{q}}\left(v_{q}-R_{s} i_{q}-\omega_{e} L_{d} i_{d}-\omega_{e} \phi_{m g}\right) \\
& \dot{\omega}_{e}=\frac{p}{J}\left(T_{e}-\frac{B}{p} \omega_{e}-T_{L}\right) \\
& T_{e}=\frac{3}{2} p\left[\phi_{m g} i_{q}+\left(L_{d}-L_{q}\right) i_{d} i_{q}\right]
\end{aligned}
$$

where the notation refers to the per unit value of a variable with the exception $\omega_{e b}$ that is in SI units. The sampling time $T_{s}$ is chosen as $100(\mu s)$ for inner loop and $200(\mu s)$ for outer loop, respectively. 
TABLE I

BASE VALUES FOR THE PER UNIT MODEL

\begin{tabular}{|l|l|l|l|}
\hline Symbol & Description & Base Value & SI unit \\
\hline$P_{b}$ & rated power & 0.35 & $\mathrm{KW}$ \\
\hline$U_{b}$ & rated voltage & $150 / \sqrt{3}$ & Volts \\
\hline$T_{b}$ & rated Torque & 1.1 & $\mathrm{Nm}$ \\
\hline$I_{b}$ & Current & 8.083 & $\mathrm{~A}$ \\
\hline$R_{b}$ & Resistance & 10.71 & $\mathrm{Ohm}$ \\
\hline$\omega_{e b}$ & velocity & 630.63 & $\mathrm{rad} / \mathrm{s}$ \\
\hline$L_{b}$ & Inductance & 0.017 & $\mathrm{H}$ \\
\hline$\Phi_{b}$ & flux & 0.1373 & $\mathrm{Webber}$ \\
\hline$J_{b}$ & inertia & 0.0018 & $\mathrm{~kg} \cdot \mathrm{m}^{2}$ \\
\hline$B_{b}$ & Viscous coefficients & 0.0018 & $\mathrm{~N} \cdot \mathrm{m} \cdot \mathrm{s}$ \\
\hline
\end{tabular}

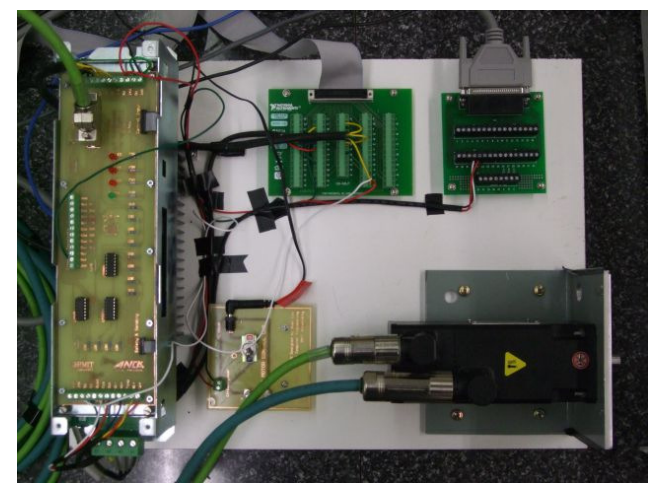

Fig. 4. PMSM test-bed

\section{B. Experiment Setups}

The experiments were conducted using the MATLAB real-time workshop and XPC host-target environment. The proposed cascade scheme was implemented in SIMULINK and downloaded into target PC. The target PC is equipped with data acquisition and quadrature encoder cards. As shown in Fig. 4, the two interface cards connected to PC are used to provide the Pulse Width Modulated (PWM) signal for the PMSM drive and obtain the feedback from encoder, respectively. The physical parameters and values for this testbed are given in Table II.

TABLE II

PARAMETERS OF PMSM

\begin{tabular}{|l|l|l|l|l|}
\hline Sym. & Description & SI Value & SI Unit & Per Unit \\
\hline $\mathrm{J}$ & total inertia & $0.47 \mathrm{e}-4$ & $\mathrm{~kg} \cdot \mathrm{m}^{2}$ & 0.0267 \\
\hline $\mathrm{B}$ & viscous coeff. & $1.1 \mathrm{e}-4$ & $\mathrm{~N} \cdot \mathrm{m} \cdot \mathrm{s}$ & 0.0625 \\
\hline$L_{d}$ & d-axis inductance & $7.0 \mathrm{e}-3$ & $\mathrm{H}$ & 0.4120 \\
\hline$L_{q}$ & q-axis inductance & $7.0 \mathrm{e}-3$ & $\mathrm{H}$ & 0.4120 \\
\hline$T_{L}$ & load torque & 0 & $\mathrm{Nm}$ & 0 \\
\hline$R_{s}$ & resistance & 2.98 & $\mathrm{Ohm}$ & 0.2781 \\
\hline$\phi_{m g}$ & $\begin{array}{l}\text { flux linkage due to } \\
\text { permanent magnet }\end{array}$ & 0.125 & Weber & 0.9102 \\
\hline$i_{\text {rated }}$ & nominal current & 2.9 & $\mathrm{Amp}$ & 0.36 \\
\hline$p$ & no. of pole pairs & 2 & & \\
\hline
\end{tabular}

\section{Results AND ANALYSis}

In this section, the results of the experimental implementation of cascade MPC to the motor considered are given and analyzed with reference to the sensitivity function (see Figure 3). The weighting matrix $R_{i}=0.01 I$ in all cases to ensure that its dynamic response is much faster than the one from the outer loop MPC.

\section{A. Zero frequency mode embedded}

For set-point following, or reference tracking, the outer loop MPC controller has to include the zero frequency. If the offset of the sensor could be accurately measured, the configuration of both inner and outer loop MPC with the zero frequency mode is sufficient for constant speed control. However, in the application, the steady-state speed will oscillate at the synchronous frequency as shown in Fig. 6. The amplitude of this oscillation is affected by the choice of weighting matrices $R_{o}$, a scalar in this case, when setting $Q=C^{T} C$ for the outer loop MPC design. From the control point of view, a large value of $R_{o}$ puts more weighting on the control input and thus slower response, which could be observed by examining the q-axis current. At the start-up, Fig. 6(b) with smaller weighting $R_{o}=1$ shows much larger transient current $(2.65 \mathrm{Amp})$ than the one $(1.3 \mathrm{Amp})$ with larger weighting $R_{o}=100$ in Fig. 5(b). From the disturbance rejection point of view, Fig. 5(a) and Fig. 6(a), a small choice of $R_{o}$ leads to less speed ripples, as validated by inspecting the input sensitivity function. Figure 3 shows that a small choice of $R_{o}$ leads to less gain for input disturbance and thus reduces the low frequency ripples to some extent.

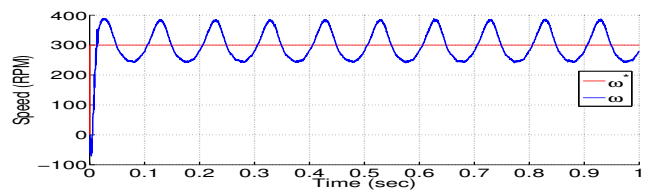

(a) Speed reference and feedback

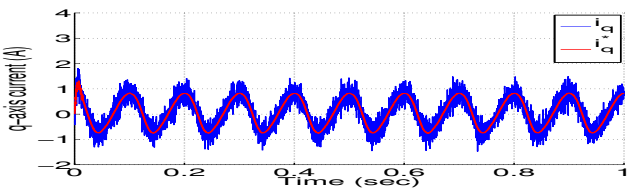

(b) q-axis current and feedback

Fig. 5. No constraints with the zero frequency embedded when $Q_{o}=C^{T} C$ and $R_{o}=100$ for outer loop MPC design.

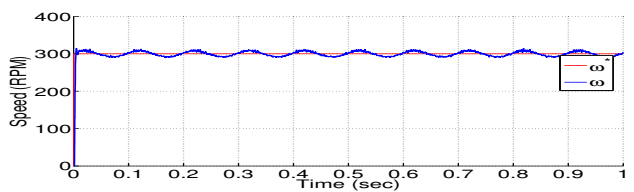

(a) Speed reference and feedback

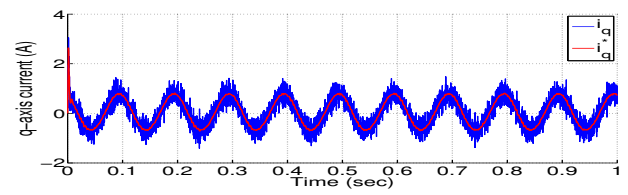

(b) q-axis current and feedback

Fig. 6. No constraints with the zero frequency embedded when $Q_{o}=C^{T} C$ and $R_{o}=1$ for the outer loop MPC design.

\section{B. Zero and 1 st frequency modes embedded}

In order to completely reject the sinusoidal disturbance, the corresponding frequency mode has to be embedded into the outer loop MPC. Moreover, the frequency of oscillation 
is fixed in the steady-state and hence the problem caused must be addressed in the steady-state. Therefore, the outer loop MPC with zero frequency is employed at the start and MPC with the zero and 1st frequency modes is switched on in the steady-state. Here the MPC structure is switched in this task. The past speed $(\omega)$ and q-axis current $\left(i_{q}\right)$ values are saved in memory to ensure bumpless transfer between two controllers as seen in the implementation equations (see (21) and (34)). Fig. 7 and 8 shows two cases where the speed ripples are minimized shortly after switching at $0.5 \mathrm{sec}$. In addition, Fig. 7(b) and 8(b) also demonstrates that the q-axis reference $i_{q}^{*}$ is constrained at 1 (Amp) when compared with Fig. 5(b) and 6(b), respectively. Those constraints could slow the speed response and provide the soft-start capability for the motor. Furthermore, it is evident from these figures that the bumpless transfer between those two controllers has been achieved in the implementation.

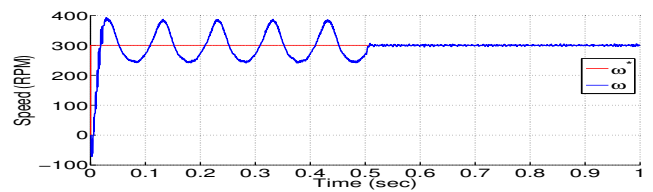

(a) Speed reference and feedback

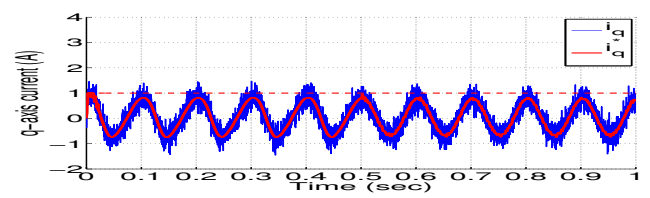

(b) q-axis current reference and feedback

Fig. 7. Constraints on q-axis reference $i_{q}^{*}$ and rejection of high magnitude ripples

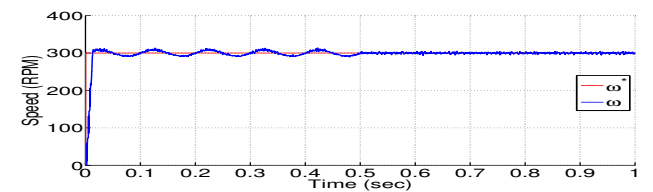

(a) Speed reference and feedback

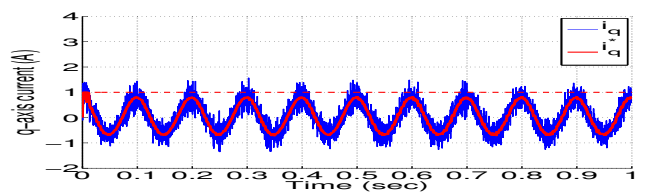

(b) q-axis current reference and feedback

Fig. 8. Constraints on q-axis reference $i_{q}^{*}$ and rejection of low magnitude ripples

\section{Robustness to frequency inaccuracy}

As observed from Fig. 3, with the 1st frequency mode included, the closed-loop gain for the input disturbance is almost zero at the corresponding frequency and greatly reduced at neighboring frequencies. Hence a frequency mode could also reduce the speed ripples at neighboring frequencies. In cases where the frequency is hard to accurately determine or varies within a certain range, the developed algorithm could also provide robustness against frequency inaccuracy.
Figure 9(a) shows a case where the frequency of ripples $(f=20 / 3 \mathrm{~Hz})$ is lower than the frequency mode $(f=10$ $\mathrm{Hz}$ ) included in the MPC design and Fig. 9(b) shows a case where the frequency of ripples $(f=20 \mathrm{~Hz})$ is higher than the frequency mode included in the MPC design. Furthermore,

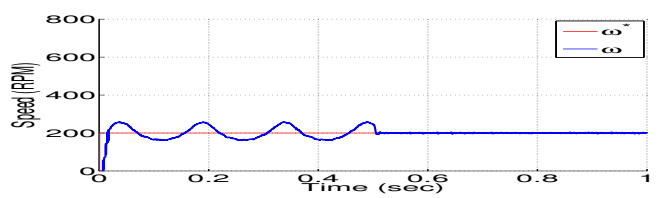

(a) robustness to low frequency ripples at 200 (RPM)

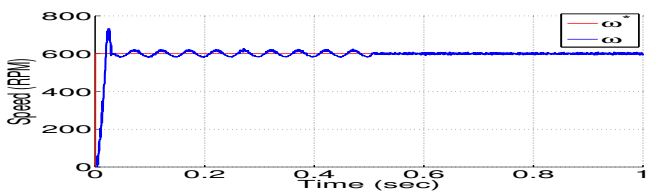

(b) robustness to high frequency ripples at 600 (RPM)

Fig. 9. Robustness with respect to frequency inaccuracy.

by taking of the advantage of the robustness to frequency inaccuracy, a single controller can be utilised to deal with speed reference variation. Fig. 10 demonstrates a case with a staircase reference, where the MPC with frequency mode $(f=20 \mathrm{~Hz})$ is switched on right after it reaches $600(\mathrm{rpm})$. Despite of variation of frequency changes, as observed in Fig. 10(b), the speed is free from oscillation.

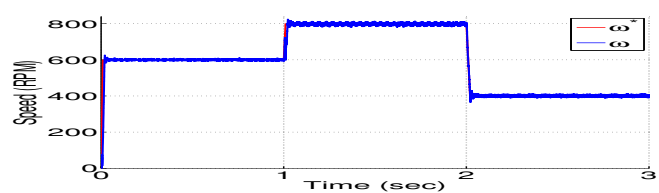

(a) speed reference and feedback

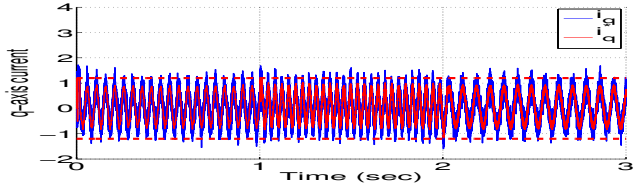

(b) q-axis current reference and feedback

Fig. 10. staircase reference with rejection of speed ripples

\section{Conclusions}

This paper has developed a cascade MPC structure for high performance speed control of a PMSM with speed ripple minimization. The inner loop MPC provides a fast feedback control to reduce the effect of disturbance and the effect of nonlinearity and model parameter uncertainty. The outer loop MPC is embedded with zero frequency mode for start-up and an extra frequency mode for minimizing the speed ripples in the steady-state operation. The MPC design is based on the per unit model of the PMSM and experimental results confirm the potential of this control scheme. It is also evident from the experimental results that smooth transition of the control signal has been achieved when the controller structure changes in real-time. 